\title{
A Method for Calculating Frame Check Sequences in Tagged Ethernet Frames
}

\author{
Hiroshi Matsuda \\ Faculty of Econoinformatic, \\ Himeji Dokkyo University \\ Hyogo, JAPAN
}

\begin{abstract}
Like other technologies such as Internet Protocol (IP) or Transmission Control Protocol (TCP), an Ethernet frame also has a field to detect some errors occurred on transmission. This field is referred to as frame check sequence (FCS). Usually, when an Ethernet frame conveys extra information on some feature such as VLAN (Virtual Local Area Network), a field called tagis inserted into the frame and FCS must be recalculated. In this paper we propose a method for calculating a frame check sequence in a tagged frame effectively. Our method utilizes the relationship between an Ethernet frame and its tagged Ethernet frame on FCS. And it is also shown that in most cases the FCS in a tagged Ethernet frame can be easily obtained by just adding the pre-calculated result to the FCS in an original Ethernet frame.
\end{abstract}

\section{General Terms}

Computer network, Error detection

\section{Keywords}

Frame Check Sequence(FCS), Virtual Local Area Network(VLAN), IEEE802.1Q

\section{INTRODUCTION}

Responding adequately to demands for enterprise networks, Ethernet has developed since its creation and become one of the essential technologies in computer networks. Note here that in this paper we denote by Ethernet a family of Ethernet-related technologies standardized by IEEE 802.3 working group. Although Ethernet has a variety of transmission media and rates, its frame format is in common use. Any Ethernet frame has the following fields except preamble:

(1) Destination Media Access Control (MAC) address: 6 bytes

(2) Source MAC address : 6 bytes

(3) Type : 2 bytes

(4) Data : 46 bytes -1500 bytes

(5) Frame Check Sequences (FCS) : 4 Bytes

FCS is a field to detect some errors occurred on transmission in the frame and is calculated based on the rest of the frame.

In addition to the increase in transmission rate, Ethernet has extended its capabilities for such features as Virtual Local Area Network(VLAN) or Class of Service(CoS). In an Ethernet frame, however, there are no optional fields like those in an Internet Protocol (IP) packet or a Transmission Control Protocol(TCP) segment. A new field,therefore, needed to be designed for extended capabilities. This new field was developed by IEEE802.1 working group and is generally referred to as IEEE 802.1Q tag.
IEEE 802.1Q tag has a length of 4 bytes and is inserted between source MAC address field and type field in a Ethernet frame when needed to convey some information on VLAN and/or CoS. At this time the FCS of the original Ethernet frame is cut off and the FCS of the tagged Ethernet frame is newly calculated. Without doubt this new FCS can be calculated by any method developed so far. It would, however, be more effective to calculate it on the basis of the relationship between the original Ethernet and its tagged Ethernet frame on FCS because they are almost the same.

In this paper we propose a method for calculating a FCS in a tagged Ethernet frame effectively. Our method utilizes the relationship between an Ethernet frame and its tagged Ethernet frame on FCS. This paper is organized as follows: Firstly, in section two we give a brief explanation on FCS and IEEE802.1Q Tag enough to understand this paper. Secondly, in section three, we propose our method and give a calculation example. And in section four we discuss our method and show that in most cases the FCS in a tagged Ethernet frame can be easily obtained by just adding the pre-calculated result to the FCS in an original Ethernet frame. Finally, in section five we summarize the results.

\section{PRELIMINARIES}

In this section we will give a brief explanation on FCS and IEEE802.1Q Tag enough to understand this paper.

\subsection{Frame Check Sequence}

Let a binary n-tuple $a_{n}=\left(a_{n-1}, a_{n-2}, \cdots, a_{1}, a_{0}\right)$ correspond to a polynomiala $(x)=a_{n-1} x^{n-1}+a_{n-2} x^{n-2}+\cdots a_{1} x+a_{0}$. And for $\mathrm{a}(\mathrm{x})$ and $\mathrm{b}(\mathrm{x})$ define the addition and multiplication respectively as follows:

$$
\begin{aligned}
& a(x)+b(x) \equiv \sum_{i=0}^{n-1}\left(a_{i} \oplus b_{i}\right) x^{i} \\
& a(x) b(x) \equiv \sum_{i=0}^{2 n-2}\left(\sum_{j=0}^{n-1} a_{j} b_{i-j}\right) x^{i}
\end{aligned}
$$

where $\oplus$ means exclusive-or and $b_{s}=0$ for $s<0$.

In an Ethernet frame its FCS is calculated based on a cyclic code [1] which is called a cyclic redundancy code(CRC). CRC has been widely used in digital communication systems to mainly detect some errors occurred on a channel. The CRC used in Ethernet is defined by the following polynomial $\mathrm{g}(\mathrm{x})$

$$
\begin{array}{r}
g(\mathrm{x})=x^{32}+x^{26}+x^{23}+x^{22}+x^{16}+x^{12}+x^{11}+x^{10} \\
+x^{8}+x^{7}+x^{5}+x^{4}+x^{2}+x+1
\end{array}
$$


that is a generator polynomial of the code. Then, if we denote by $\mathrm{m}(\mathrm{x}) \mathrm{a}$ polynomial that corresponds to a binary sequence made by removing its FCS portion from an Ethernet frame, a polynomial $f(x)$ that corresponds to the FCS portion is given by the following equation.

$$
f(\mathrm{x})=m(x) x^{32} \bmod g(\mathrm{x})
$$

where "A mod B" means the remainder when A is divided by B.In this case, therefore, the polynomial that corresponds to all the bits in the Ethernet frame amounts to the polynomial

$$
m(\mathrm{x}) x^{32}+f(x) .
$$

In many areas where CRC has been used how fast the encoding or decoding of CRC can be done is one of the most important points and the method on it has been under intense investigation[2-6].

\subsection{IEEE802.1Q Tag}

Suppose that four PCs are connected to two switches as shown in Fig.1.

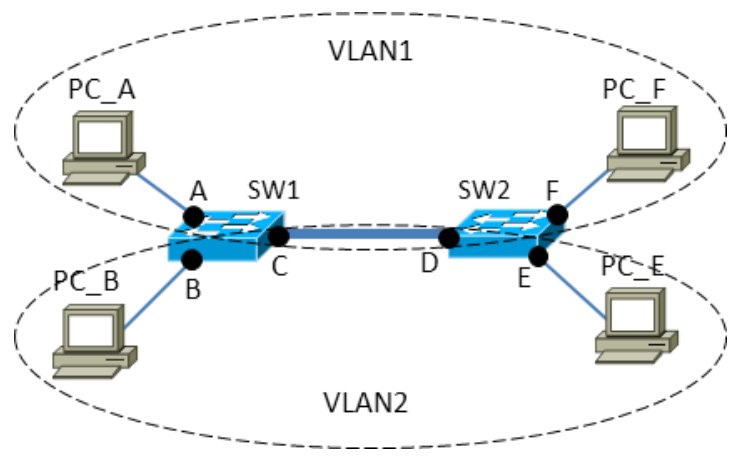

Fig.1 : Two VLANs in a switched network

All of the ports in a switching hub usually belong to the same broadcast domain. Setting Virtual Local Area Network(VLAN) [7], however, enables us to separate a broadcast domain into some narrower ones.In Fig.1 port $\mathrm{A}, \mathrm{C}, \mathrm{D}$ and $\mathrm{F}$ belong to VLAN1 and port $\mathrm{B}, \mathrm{C}, \mathrm{D}$, and $\mathrm{E}$ belong to VLAN 2.Note here that a port can belong to some VLANs. In this case a broadcast frame sent by PC A must reach port $\mathrm{F}$ but not reach port Ein SW2. To this end SW2 must distinguish what VLAN a received frame belongs to. IEEE 802.1Q [8] is one of the ways to give the ability to switching hubs. This standardized techniqueexpands existing Ethernet frame format to include extra information such as VLAN number. This new field where the extra information is packed is referred to as a tag and placed between source address field and type field in an Ethernet frame shown as Fig.2. In a tag VLAN Identifier(VID) field includes the VLAN number an original frame belongs to. The length of a tag is only four bytes.

\begin{tabular}{|l|l|l|l|l|}
\hline D.A. & S.A. & TYPE & data & FCS \\
\hline
\end{tabular}

Fig. 2 : IEEE 802.1Q Tag
When a switching hub makes a tagged frame, at first a tag is inserted into an original frame and the FCS of anoriginal frame is removed and then a new FCS of the tagged frame is calculated in the same manner as other frames. However, considering a tagged frame is almost the same as an original frame because the length of a frame is between 64 bytes and 1518 bytes, it is thought effective to utilize the relationship between the frames for calculating a FCS in a tagged frame.

In the next section we propose a new method for calculating a FCS in a tagged frame through the use of the knowledge of an original frame.

\section{CALCULATING METHOD}

Firstly we define some polynomials to decompose a tagged frame(Fig.3).

\begin{tabular}{c|c|c|c|}
\multicolumn{2}{c|}{$h(X)$} & $d(X)$ & $f_{\text {org }}$ \\
\hline$h(X)$ & $0 \cdot 0$ & $d(X)$ & $f_{h}$ \\
\hline \multicolumn{3}{|c|}{} \\
\cline { 2 - 4 } & $v(X)$ & $0 \cdot 0$ & $f_{v}$ \\
\hline
\end{tabular}

Fig. 3 : Decomposition of a tagged frame

Leth $(x), d(x)$, and $f_{\text {org }}(x)$ be the polynomial expressing the bits ranging from the left most of the frame to the rightmost of source address field in a frame, the polynomial expressing the bits ranging from the leftmost of type field to the rightmost of data field in a frame, and the polynomial expressing the bits corresponding to FCS field,respectively. Then, an original frame can be expressed as the following polynomial:

$$
h(\mathrm{x}) \mathrm{x}^{8(\mathrm{~L}+4)}+d(\mathrm{x}) \mathrm{x}^{8 \cdot 4}+f_{\text {org }}(\mathrm{x})
$$

where L means the highest order of $\mathrm{d}(x)$ plus one. And by (1)

$$
f_{\text {org }}(\mathrm{x})=\left\{h(\mathrm{x}) \mathrm{x}^{8(\mathrm{~L}+4)}+d(\mathrm{x}) \mathrm{x}^{8 \cdot 4}\right\} \bmod g(\mathrm{x})
$$

Now consider the following polynomials:

$$
\begin{gathered}
h(\mathrm{x}) \mathrm{x}^{8(\mathrm{~L}+8)}+d(\mathrm{x}) \mathrm{x}^{8 \cdot 4}+f_{h}(\mathrm{x}) \\
v(\mathrm{x}) \mathrm{x}^{8(\mathrm{~L}+4)}+f_{v}(\mathrm{x})
\end{gathered}
$$

where $\mathrm{v}(\mathrm{x})$ means the polynomial expressing the bits corresponding to tag field and

$$
\begin{gathered}
f_{h}(\mathrm{x}) \equiv\left\{h(\mathrm{x}) \mathrm{x}^{8(\mathrm{~L}+8)}+d(\mathrm{x}) \mathrm{x}^{8 \cdot 4}\right\} \bmod g(\mathrm{x}) \\
f_{v}(\mathrm{x}) \equiv v(\mathrm{x}) \mathrm{x}^{8(\mathrm{~L}+4)} \bmod g(\mathrm{x})
\end{gathered}
$$

As shown in Fig.3,then, the polynomial expressing the bits corresponding to FCS field of a tagged frame, which is denoted by $f_{\operatorname{tag}}(x)$, meets the equation

$$
f_{\text {tag }}(x)=f_{h}(x)+f_{v}(x)
$$

Combining (4) with (5) and (6) gives

$$
\begin{aligned}
f_{\text {org }}(\mathrm{x}) & +f_{h}(x)+f_{v}(x) \\
= & \left\{h(x)+v(x)+h(x) x^{8 \cdot 4}\right\} x^{8(L+4)} \bmod g(x)
\end{aligned}
$$

Therefore, we have

$$
f_{\text {tag }}(x)=f_{\text {org }}(x)+f_{\text {dif }}(x)
$$


where

$$
f_{\text {dif }}(x) \equiv\left\{h(x)+v(x)+h(x) x^{8 \cdot 4}\right\} x^{8(L+4)} \bmod g(x)(10)
$$

Both (9) and (10)imply that a FCS in a tagged frame depends not on the content in data field,but on the length of data field.

Since the degree of $\mathrm{h}(\mathrm{x})$ is less than or equal to $8 \times 11=$ 88we define $\left\{\mathrm{C}_{\mathrm{i}}(\mathrm{x})\right\}(\mathrm{i}=0,1, \cdots, 15)$ by the following equation

$$
\sum_{i=0}^{15} C_{i}(x) \cdot x^{8 i} \equiv h(x)+v(x)+h(x) x^{8 \cdot 4}
$$

where the degree of $C_{i}(x)$ is less than or equal to 7.So, from (10) we have

$$
f_{\text {dif }}(x)=\sum_{\mathrm{i}=0}^{15} C_{i}(x) \cdot R_{i}(\mathrm{x}) \bmod g(x)
$$

where

$$
R_{i}(x)=x^{8(L+4+i)} \bmod g(x)(i=0,1, \cdots, 15)
$$

It follows that a FCS in a tagged frame can be effectively calculated by parallel computation provided that we would have precalculated results of all of the right-hand membersin (12). Since the length $L$ is between 48 and 1502 the table that has all of the precalculated results of them requires about $6 \mathrm{~K}$ bytes and so is easily implementable. As we will state later it is, of course, still effective to have a smaller table: The size of the table depends on some conditions such as cost or permissible time to calculate FCS.

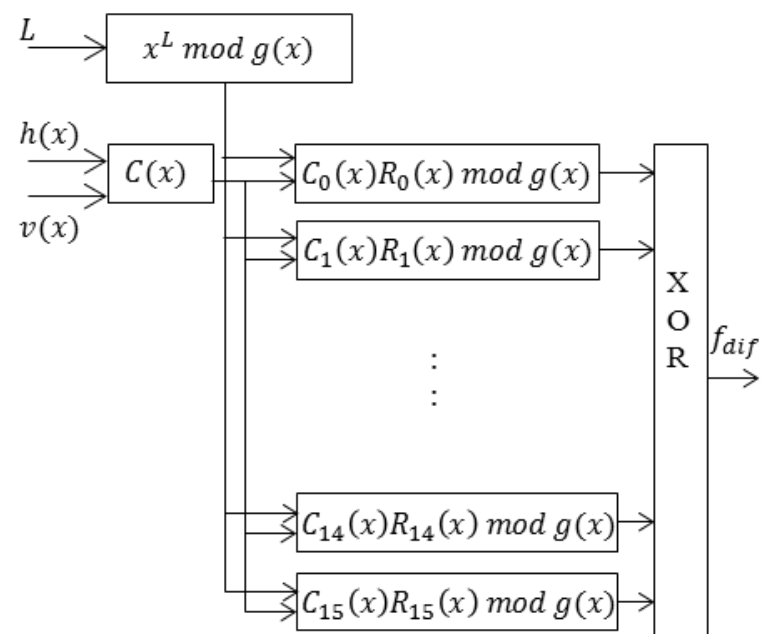

Fig. 4: Conceptual model of parallel computation

In Fig. 4 we show a conceptual model of our method.

\section{EXAMPLE CALCULATION AND DISCUSSIONS}

Consider the example network in Fig.5. SW has some ports belonging to different VLANs and Port A belongs to VLAN 5.In such a situation SW inserts an IEEE 802.1Q tag into any frame transmitted to Router since Router must distinguish what VLAN a received frame belongs to.

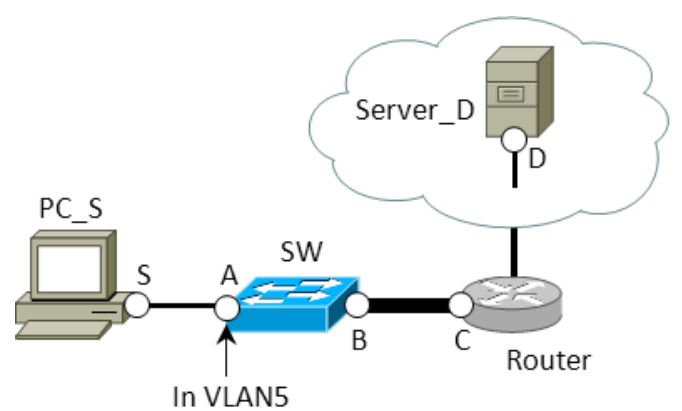

Fig. 5 : Sample network forwarding tagged frames

Now suppose that PC_Ssends the following frame F1, which has the lowest priority, to Server_D.Note here that the binary sequence including F1 is given in hexadecimal notation from the leftmost of the sequence.

\section{F1: 78 bytes}

00 0d 0b b5 8b 4888 ae 1d 28 3b 470800450000 3c 46 3b $000080010000 \mathrm{c} 0 \mathrm{a} 80 \mathrm{~b} 03$ ca d6 ca $6508004 \mathrm{~d} 560001$ $00056162636465666768696 \mathrm{a} 6 \mathrm{~b}$ 6c 6d 6e 6f 707172 7374757677616263646566676869 a3 4a ba 29

We,therefore,can break this frame into its constituent $\operatorname{partsh}(x), d(x)$ and $f_{\text {org }}(x)$ as follows.

\begin{tabular}{|c|c|c|}
\hline$i$ & $C_{i}(x)$ & $C_{i}(x) R_{i}(x) \bmod g(x)$ \\
\hline 0 & 42 & $\begin{array}{llllllll}2 & 5 & 6 & 4 & 0 & 7 & 3 & 1 \\
\end{array}$ \\
\hline 1 & $1 \mathrm{~b}$ & $\begin{array}{llllllll}5 & 7 & 4 & 7 & \text { a } & 9 & 0 & 0\end{array}$ \\
\hline 2 & 28 & 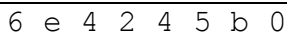 \\
\hline 3 & $9 \mathrm{C}$ & 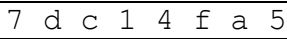 \\
\hline 4 & e 9 & $\begin{array}{llllllll}1 & 6 & 3 & 1 & 1 & 2 & 2 & 6\end{array}$ \\
\hline 5 & b 3 & 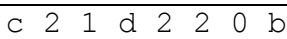 \\
\hline 6 & 60 & $\begin{array}{llllllll}\mathrm{d} & 3 & \mathrm{c} & 0 & 3 & 1 & \mathrm{c} & 9\end{array}$ \\
\hline 7 & 96 & 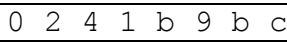 \\
\hline 8 & $1 \mathrm{~b}$ & $\begin{array}{llllllll} & 0 & d & d & 7 & 5 & d & a\end{array}$ \\
\hline 9 & 83 & 5 a d 399 a f \\
\hline 10 & 45 & a 37 f a 9 d 9 \\
\hline 11 & $8 \mathrm{~b}$ & e 33 e 29 c 7 \\
\hline 12 & b 5 & 9 e 788 d d f \\
\hline 13 & $0 \mathrm{~b}$ & $\begin{array}{llllllll}2 & \mathrm{C} & 7 & 4 & 5 & 2 & \mathrm{c} & \mathrm{a}\end{array}$ \\
\hline 14 & $0 \mathrm{~d}$ & c $\quad 6$ a 0 c c $\quad 6$ a 6 \\
\hline 15 & 00 & $\begin{array}{llllllll}0 & 0 & 0 & 0 & 0 & 0 & 0 & 0\end{array}$ \\
\hline \multicolumn{2}{|c|}{$f_{\text {dif }}(x)$} & $\begin{array}{llllllll}0 & 7 & 3 & 4 & 4 & 1 & d & 9 \\
\end{array}$ \\
\hline \multicolumn{2}{|c|}{$f_{\text {org }}(x)$} & $\begin{array}{llllllll}\mathrm{a} & 3 & 4 & \mathrm{a} & \mathrm{b} & \mathrm{a} & 2 & 9\end{array}$ \\
\hline \multicolumn{2}{|c|}{$f_{\text {tag }}(x)$} & $\begin{array}{llllllll}\mathrm{a} & 4 & 7 & \mathrm{e} & \mathrm{f} & \mathrm{b} & \mathrm{f} & 0\end{array}$ \\
\hline
\end{tabular}

$h(x): 12$ bytes

00 0d 0b b5 8b 4888 ae 1d 28 3b 47

Table 1: An Example calculation of FCS in a tagged frame

$d(x): 62$ bytes $(\mathrm{L}=62)$

$08004500003 \mathrm{c} 46$ 3b $000080010000 \mathrm{c} 0$ a8 $0 \mathrm{~b} 03$ ca d6 ca 6508004 d $56000100056162636465666768696 \mathrm{a}$ 6b 6c 6d 6e 6f 707172737475767761626364656667 6869

$f_{\text {org }}(x): 4$ bytes

a3 4 a ba 29

And since Priority Code Point (PCP) in the tag is one,

$v(x): 4$ bytes

81002005 
Substituting $h(x)$ and $v(x)$ into (11) we obtain $f_{\text {dif }}(x)$ and,consequently, $f_{\text {tag }}(x)$ from (9) in Table 1.

The configuration used in this sample network in Fig.5 is very common among actual LANs: A host such as a PC or a printer has a dedicated port in the nearest switching hub to it and a VLAN number is assigned to such a port. In addition a group of servers is typically placed in other VLANs than any VLANs used for end users: This means that most of the communications end users begin require routing considering that each VLAN has its own subnet. For the frames used in such a communication,therefore, both source MAC address(at PC) and destination MAC address (at Router) are invariant like the VLAN assigned to each port. This means that $f_{\text {tag }}(x)$ can be easily obtained by one XOR operation between $f_{\text {org }}(x)$ and $f_{\text {dif }}(x)$ once $f_{\text {dif }}(x)$ is given. In comparison with other methods it is desirable that this method doesn't depend on frame length in calculation time.

In Table 2 we show examples of FCSs in tagged frames with typical lengths when PC_S sends frames destined for any host beyond Router in Fig.5. Noticehere that L is between 48(minimum data size 46 plus type field size 2) and 1500 (maximum data size 1500 plus type field size 2) in bytes. And the last two lengths 202 and 62 in Table 2 are both based on Voice over IP(VoIP): The former is equal to G.711 data size 202 plus type field 2 in bytes and the latter G.729 data size 60 plus type field 2 in bytes, respectively [9].Since severe restrictions on delayare imposed on VoIP our method is particularly effective to shorten time necessary to calculate FCSs in such a situation.

Table 2: Examples of FCSsin tagged frames withtypical Lengths

\begin{tabular}{|c|c|c|c|}
\hline $\mathrm{L}$ & $f_{\text {dif }}$ & $\mathrm{L}$ & $f_{\text {dif }}$ \\
\hline \hline 48 & $73 d 1 \mathrm{c} 808$ & 1026 & $75649 \mathrm{~b} 0 \mathrm{~d}$ \\
\hline 130 & $\mathrm{~b} 9404234$ & 1502 & $9 \mathrm{c} 56903 \mathrm{f}$ \\
\hline 258 & $03626 \mathrm{~d} 58$ & 202 & $9838 \mathrm{e} 473$ \\
\hline 514 & $\mathrm{~b} f \mathrm{c} 7 \mathrm{~b} 084$ & 62 & $073441 \mathrm{~d} 9$ \\
\hline
\end{tabular}

\section{CONCLUSIONS}

In this paper we have proposed a method for calculating a FCS in a tagged Ethernet frame effectively. Our method utilizes the relationship between an Ethernet frame and its tagged Ethernet frame on FCS because those frames are much of the same. Considering that most of switching hubs have their ports preassigned a VLAN number and connecting only a host, our method is capable of wide application to make time necessary to obtain a FCS in a tagged frame shorten. In particular, it is more useful to use our method in a situation such as VoIP or teleconference systems that has a little tolerance to time delay. Simplifying parallel computation in our method will be one of the future works.

\section{REFERENCES}

[1] F.J.Macwilliams, N.J.A.Sloane, The Theory of ErrorCorrecting Codes, North-Holland, NETHERLANDS, 1992.

[2] H.H. Mathukiya, N.M. Patel," A Novel Approach for Parallel CRC Generation for High Speed Application", 2012 International Conference on Communication Systems and Network Technologies (CSNT), pp.581 585,2012

[3] J.Peng, Y. Zhou,Y.Yang," Cyclic redundancy code check algorithm based on small lookup table", IEEE International Conference on Communications Technology and Applications, pp.596- 599, 2009

[4] T.Henriksson, H.Eriksson, U.Nordqvist, P.LarssonEdefors, D.Liu, "VLSI implementation of CRC-32 for 10 Gigabit Ethernet", Proceedings of 2001 IEEE 8th IEEE International Conference on Electronics, Circuits and Systems, vol.3, pp. 1215-1218, 2001

[5] G.Albertengo, R.Sisto, "Parallel CRC generation", IEEE Micro, vol.10, no.5, pp.63-71, 1990.

[6] T.V.Ramabadran, S.S.Gaitonde, "A tutorial on CRC computations", IEEE Micro, vol.8, no.4, pp.62-75, 1988.

[7] Online Available IEEE802.3-2008

http://standards.ieee.org/getieee802/download/802.32008_section1.pdf

[8] Online Available IEEE802.1Q-2011 - IEEE Standard for Local and metropolitan area networks--Media Access Control (MAC) Bridges and Virtual Bridged Local Area Networkshttp://standards.ieee.org/findstds/standard/802. 1Q-2011.html

[9] Online Available

"Voice Over IP - Per Call BandwidthConsumption" : http://www.cisco.com/en/US/tech/tk652/tk698/technolog ies_tech_note09186a0080094ae2.shtml 